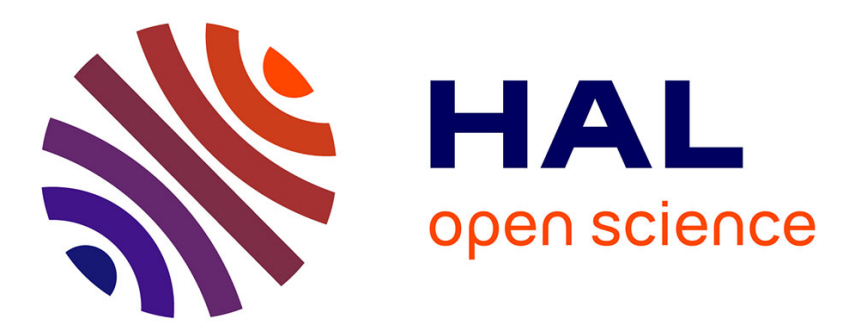

\title{
A three-dimensional dissipative map modeling type-II intermittency
}

\author{
Françoise Argoul, A. Arneodo, P. Richetti
}

\section{To cite this version:}

Françoise Argoul, A. Arneodo, P. Richetti. A three-dimensional dissipative map modeling type-II intermittency. Journal de Physique, 1988, 49 (5), pp.767-775. 10.1051/jphys:01988004905076700 . jpa-00210753

\section{HAL Id: jpa-00210753 https://hal.science/jpa-00210753}

Submitted on 1 Jan 1988

HAL is a multi-disciplinary open access archive for the deposit and dissemination of scientific research documents, whether they are published or not. The documents may come from teaching and research institutions in France or abroad, or from public or private research centers.
L'archive ouverte pluridisciplinaire HAL, est destinée au dépôt et à la diffusion de documents scientifiques de niveau recherche, publiés ou non, émanant des établissements d'enseignement et de recherche français ou étrangers, des laboratoires publics ou privés. 


\title{
A three-dimensional dissipative map modeling type-II intermittency
}

\author{
F. Argoul $\left({ }^{*}\right)$, A. Arneodo (**) and P. Richetti $\left({ }^{1}\right)$ \\ Center for Nonlinear Dynamics, Physics Department, University of Texas, Austin, TX 78712, U.S.A. \\ ( $\left.{ }^{1}\right)$ Centre de Recherche Paul Pascal, Domaine Universitaire, 33405 Talence Cedex, France
}

(Reçu le 22 juillet 1987, accepté sous forme définitive le 4 janvier 1988)

\begin{abstract}
Résumé. - Pour décrire la transition vers le chaos par intermittence de type II, nous proposons une application de Poincaré modèle à trois dimensions qui rend compte de l'interaction entre une bifurcation locale de Hopf souscritique et une bifurcation globale homocline. Une telle description, parce qu'elle se veut de dimension minimale, affecte le degré de stabilité structurelle de ce scénario et nécessite l'adaptation du formalisme de Pomeau-Manneville au cas d'un mécanisme de réinjection homocline unidimensionnel. Nous présentons les résultats de simulations numériques qui confirment les prédictions théoriques et ce aussi bien pour les distributions statistiques des longueurs des plages laminaires que pour la divergence en $1 / f^{\Delta}$ des spectres de puissance au voisinage du seuil de transition. Nous discutons ensuite l'influence d'un bruit aléatoire sur le scénario d'intermittence de type II en distinguant les cas de bruits additifs et multiplicatifs.
\end{abstract}

\begin{abstract}
A three-dimensional Poincaré map model for type-II intermittency is proposed. The purpose is to describe this transition in minimal dimension which in turn affects the degree of structural stabilit; of the scenario. This scenario is understood in terms of the interaction of a local subcritical Hopf bifurcation and a global homoclinic bifurcation. The Pomeau-Manneville picture is revisited in order to account for the onedimensional homoclinic reinjection process. The distribution of the lengths of the laminar episodes is investigated and numerical results are shown to corroborate the theoretical predictions. The influence of noise on type-II intermittency is discussed. The difference between additive and multiplicative noise is emphasized.
\end{abstract}

\section{Introduction.}

The introduction by Pomeau and Manneville [1] of the notion of intermittency as a specific route to weak turbulence has stimulated many theoretical [2, 3], numerical [4-6] and experimental [7-11] studies during the past few years. However, in most of these studies, and mainly in the experimental approaches, the emphasis has been on the analysis of intermittent regimes and not on a detailed investigation of the nature of the transition to chaos. In fact, for intermittency to be a second-order phase transition

$\left(^{*}\right)$ Permanent address : Centre de Recherche Paul Pascal, Domaine Universitaire, 33405 Talence Cedex, France.

(**) Permanent address: Laboratoire de Physique Théorique, Université de Nice, Parc Valrose, 06034 Nice Cedex, France. from ordered to disordered temporal patterns, two ingredients must actually occur simultaneously : (i) a local instability of a limit cycle which can be either a saddle-node bifurcation (Type-I intermittency), or a subcritical Hopf bifurcation (Type-II intermittency), or a subcritical period-doubling bifurcation (TypeIII intermittency); and (ii) a global nonlinear mechanism, e.g. strange-attractor-like behavior, which ensures the reinjection of the dynamics in the neighborhood of the limit cycle. Then, when varying a control parameter, a continuous transition is observed from a periodic regime to short turbulent bursts interrupting (seemingly at random) nearly periodic oscillations. This legitimizes the definition of critical exponents which characterize the evolution of the distribution of laminar episodes. As far as type-I [12] and type-III [13] intermittencies are concerned, it has been shown that not only the local instability but also the reinjection mechanism 
strongly condition the statistics of the laminar regions. One of the issues of this paper, which is devoted to the study of type-II intermittency, is to point out that the nature of the reinjection process may even affect the degree of structural stability [14] of this scenario to chaos.

Among the three types of intermittency originally defined by Pomeau and Manneville [1], type-II intermittency is undoubtedly the least popular, since for years there have been no examples identifying this route in either real experiments or in simulation studies. In fact only formal models of type-II intermittency have been proposed. These models are essentially based on the subcritical Hopf normal form [15] for mappings :

$$
\left\{\begin{array}{l}
\rho^{\prime}=(1+\varepsilon) \rho+a \rho^{3}+\text { h.o.t. } \\
\theta^{\prime}=\theta+\Omega+b \rho^{2}+\text { h.o.t. }
\end{array}\right.
$$

together with an artificially added reinjection mechanism obtained by periodizing the phase space or by assuming a uniform random reinjection distribution $P_{\mathrm{r}}\left(\rho_{\mathrm{r}}\right)=1 / \pi R_{\mathrm{r}}^{2}$ in a disk of radius $R_{\mathrm{r}}\left(\rho_{\mathrm{r}}<R_{\mathrm{r}}\right)$ contained in the two-dimensional unstable manifold of the fixed point $(\rho=0)$. With this working hypothesis and according to the rotational invariance of (1), the reentry points are chosen at random with uniform probability in this disk. Generally, $R_{\mathrm{r}} \leqslant R_{\ell}$ where $R_{\ell}$ delimits the laminar episodes. For the sake of simplicity, Pomeau and Manneville [1] have considered the extreme case $R_{\mathrm{r}}=R_{\ell}$. The probability distribution $P_{\ell}(n)$ of the laminar lengths ( $n$ is the number of iterates) is then predicted to scale like $P_{\ell}(n) \sim n^{-2}$ at small laminar period $n$ and to decay exponentially $P_{\ell} \sim \exp (-2 \varepsilon n)$ at large $n$. In addition a straightforward calculation yields :

$$
\langle n\rangle \sim \frac{1}{2 a R_{\ell}^{2}} \ln \left(1+a R_{\ell}^{2} / \varepsilon\right)
$$

which implies that the mean length of the laminar episodes behaves like :

$$
\langle n\rangle \sim \ln (1 / \varepsilon) \quad \text { if } \quad \varepsilon \ll a R_{\ell}^{2} .
$$

At this point let us mention that a cross-over phenomenon is expected to occur at higher values of $\varepsilon$ where $\langle n\rangle$ is predicted to scale like :

$$
\langle n\rangle \sim \ln (\varepsilon) / \varepsilon \quad \text { if } \quad \varepsilon \gg a R_{\ell}^{2} .
$$

In a recent publication [16], we have reported on the first numerical observation of type-II intermittency in a nonautonomous differential system. This system is a periodically driven third-order nonlinear oscillator :

$$
\begin{aligned}
& \dddot{X}+\eta \ddot{X}+\nu \dot{X}+\mu X+k_{1} X^{2}+k_{2} \dot{X}^{2}+ \\
& +k_{3} X \dot{X}+k_{4} X \ddot{X}+k_{5} X^{2} \ddot{X}=F \cos \omega t
\end{aligned}
$$

which in the absence of forcing $(F=0)$ accounts for the interaction of a (local) subcritical Hopf bifurcation [17-19] and a (global) Shil'nikov [20] type homoclinic bifurcation. These conditions taken together favor a chaotic reinjection of the dynamics in the neighborhood of the origin and provide a nice description of an intermittent transition from a steady state to a turbulent regime consisting of chaotic bursts which from time to time emerge from a nearly stationary signal. When the periodic forcing is turned on $(F \neq 0)$, the phase space of the dynamical system (5) enlarges from $R^{3}$ to $R^{3} \times T$ and this transition to chaos generalizes to type-II intermittency. A steady state in the absence of forcing becomes a periodic orbit and the intermittent signal transforms into chaotic bursts which interrupt a nonsaturated periodic modulation of the amplitude of the original oscillation [16].

The aim of this paper is to construct a threedimensional dissipative map which models the Poincaré map of the oscillator (5) as numerically obtained in reference [16] when sampling the orbit in phase space at the frequency of the external forcing. In the neighborhood of the subcritical Hopf bifurcation, this mapping will reduce to the two-dimensional Hopf normal form (1) and only one direction will remain in which the homoclinic process can reinject the dynamics near the fixed point. In this sense, the three-dimensional discrete system defined in section 2 will provide a description of type-II intermittency in minimal dimension. In our preliminary numerical study [16] of this scenario with the differential system (5), we have failed to measure accurately critical exponents because this estimate would have required prohibitive time consuming simulations. In this new analysis, we will take advantage of the iterative nature of our dynamical system to compute the statistical distribution of the laminar episodes. In particular we will emphasize that the homoclinic reinjection mechanism is one-dimensional (and not two-dimensional) and that the theoretical results (2), (3) and (4) need to be reconsidered. In section 3 , we will revisit these predictions and produce numerical outcomes which corroborate our analytical results. Moreover we will point out that for this transition to be continuous one must define an experimental path which crosses the hypersurface of codimension 2 where both the subcritical Hopf bifurcation and the homoclinic bifurcation occur simultaneously. In other words, type-II intermittency will appear as a scenario whose degree of structural stability [14] is $d_{\mathrm{s}}=2$, unlike the original picture of Pomeau-Manneville [1] where the three types of intermittency have a degree of structural stability $d_{\mathrm{s}}=1$. Furthermore, we will elaborate on the fuzziness of this hypersurface which will allow us to conclude that the degree of structural stability of this scenario to chaos is actually $d_{\mathrm{s}}=" 2-\varepsilon "$. Finally 
we will discuss in section 3 the robustness of type-II intermittency to the presence of noise, considering both the additive and multiplicative cases.

\section{A Poincaré map model for type-II intermittency : construction and simulation.}

2.1 Construction. - In a previous work [21], one of us (A. A.) has already contributed to the construction of a three-dimensional Poincaré map model for the periodically forced oscillator (5). The following families of diffeomorphisms of $R^{3}$ have been proposed :

$$
\left\{\begin{aligned}
x^{\prime}= & x+y \\
y^{\prime}= & y+z \\
z^{\prime}= & (1-\eta) z-\nu y-\mu x-k_{1} x^{2}-k_{2} y^{2}- \\
& -k_{3} x y-k_{4} x z-k_{5} x^{2} z
\end{aligned}\right.
$$

which preserve the triple degeneracy (three Floquet multipliers equal to unity) of the origin at $\mu=$ $\nu=\eta=0$ as implemented in the unforced $(F=0)$ three dimensional oscillator (5). In the neighborhood of this tricritical (hyper) surface [22], $\mu, \nu$ and $\eta$ are small and we can introduce the scaled variables:

$$
\begin{aligned}
x & =\delta^{3} X, & y=\delta^{4} Y, & z=\delta^{5} Z, \\
\eta & =\delta \bar{\eta}, & \nu=\delta^{2} \bar{\nu}, & \mu=\delta^{3} \bar{\mu},
\end{aligned}
$$

where $\delta$ is a small positive number. Then the diffeomorphism (6) becomes a map close to the identity [23]

$$
\left\{\begin{aligned}
X^{\prime}= & X+\delta Y \\
Y^{\prime}= & Y+\delta Z \\
Z^{\prime}= & Z-\delta\left[\bar{\eta} Z+\bar{\nu} Y+\bar{\mu} X+k_{1} X^{2}+\delta k_{3} X Y+\right. \\
& \left.+\delta^{2} k_{2} Y^{2}+\delta^{2} k_{4} X Z+\delta^{5} k_{5} X^{2} Z\right] .
\end{aligned}\right.
$$

As long as $\delta$ is not too large, we can approximate $\left(X^{\prime}-X\right) / \delta,\left(Y^{\prime}-Y\right) / \delta$ and $\left(Z^{\prime}-Z\right) / \delta$ by derivatives and the mapping (8) by a system of firstorder differential equations which up to leading order in $\delta$, is equivalent to the unforced threedimensional oscillator (5) with only $X^{2}$ as nonlinear quadratic term :

$$
\dddot{X}+\bar{\eta} \ddot{X}+\bar{\nu} \dot{X}+\bar{\mu} X+k_{1} X^{2}=0 .
$$

Since such a system has been pointed out to be a paradigm for the cascade of period-doubling bifurcations [22] (Eq. (9) is the asymptotic normal form of the $\zeta^{3}$ instability), it must be emphasized that arbitrarily close $(\delta \mapsto 0)$ to the tricritical surface $\mu=\nu=\eta=0$, the three-dimensional dissipative map (6) displays the cascade of period-doubling invariant tori $[21,24]$.
Unfortunately, as discussed in reference [16], the asymptotic normal form (9) does not account for the interaction of a subcritical Hopf bifurcation and a Shil'nikov homoclinic bifurcation. This concomitant situation requires the presence of other quadratic terms in the unforced third-order oscillator (5). This implies that the Poincaré map model (6) has to be maintained at some distance from the tricritical surface $\mu=\nu=\eta=0$ (as characterized by small but finite values of $\delta$ ) in order to be suited to provide a local description of type-II intermittency. As noted by Arnold [25] in the study of some codimensiontwo bifurcations of periodic orbits, when the distance to the polycritical condition is increased, the differential system derived as just explained no longer approximates the mapping for long times. Then one expects to observe some deviations from the dynamics predicted by the continuous system (9), eventhough one takes into account higher order terms in $\delta$ (e.g. the missing quadratic terms). Therefore a specific analysis of the Poincaré map model (6) is needed to locate the Hopf and homoclinic bifurcations. When linearizing the mapping (6) around the origin, a straightforward calculation yields the following condition for this fixed point to become unstable through a Hopf bifurcation :

$\mu=\left[-(1+\eta-2 \nu)+\sqrt{(1+\eta)^{2}-4 \nu}\right] / 2$.

Let us note that equation (10) reduces to the condition [16] $\mu=\eta \nu$ in the limit $\delta \mapsto 0$ along the asymptotic path (7). In the neighborhood of the critical surface defined by equation (10), the use of both the center manifold theorem and the normal form techniques $[15,19,25]$ allows us to reduce the Poincaré map model (6) to the normal form (1) of the Hopf bifurcation for mappings. In equation (1), $\varepsilon$ measures the distance to the critical surface, while the coefficients $a$ and $b$ are computed on this surface. Although the detailed expressions of these coefficients as functions of the parameters $k_{i}$ of the nonlinear terms in the mapping (6) are rather complicated, let us mention that the arbitrariness in the $k_{i}$ covers both the situations $a>0$ and $a<0$, which correspond to subcritical and supercritical Hopf bifurcations respectively.

To locate Shil'nikov (spiraling out) homoclinic orbits in ordinary differential equations, Gaspard and Nicolis $[26,27]$ have developed a numerical technique which consists in (i) studying the intersection of the two-dimensional unstable manifold of the saddle focus with a plane which is transverse to the local one-dimensional stable manifold ; this intersection is generated when considering several trajectories starting from the local unstable manifold near the singular point; (ii) calculating analytically the trace of the one-dimensional stable manifold in this plane ; (iii) using a trial and error method to deter- 
mine the condition at which the unstable manifold contains the stable one. This technique can be used to define codimension-one hypersurfaces in the parameter space of the continuous system (9) where such homoclinic bifurcations occur. Since in the limit $\delta \mapsto 0$, the asymptotic normal form (9) provides a good approximation of the Poincaré map model (6), one can adapt this numerical technique to locate homoclinic orbits in discrete systems. Then in nearly homoclinic conditions, one may expect to find chaos as ensured by a theorem by Shil'nikov [20]. But one has to be very careful because for finite values of $\delta$, this research becomes rather tricky since one expects the homoclinic connection to split due to transverse intersections of the unstable and stable manifolds of the saddle focus. Henceforth there is no longer a well defined codimension-one homoclinic hypersurface in the parameter space but instead a fuzzy region around this hypersurface where this new source of chaos may lead to hyperchaos [28] with two positive Lyapunov exponents : one positive Lyapunov exponent corresponding to Shil'nikov homoclinic chaos, the second one resulting from the creation of subsequent Smale horseshoes [29] via the transverse intersections of the invariant manifolds of the fixed point. Let us mention that the width of this fuzzy region enlarges from zero when $\delta$ is increased.

Here, our goal is to trace the homoclinic bifurcation i.e. the corresponding codimension " $1-\varepsilon$ " fuzzy region, up to a close neighborhood of the previously described subcritical Hopf bifurcation. In this way we will approximately define the codimension " $2-\varepsilon$ " fuzzy hypersurface that our one-parameter numerical path will have to cross for type-II intermittency to be observed when iterating the Poincaré map model (6). In that sense, the degree of structural stability [14] of this scenario to chaos is $d_{\mathrm{s}}=" 2-\varepsilon "$. At this point, let us mention that, when increasing $\delta$ in equation (7), higher order nonlinear terms such that $k_{5} X^{2} Z$ are needed in the discrete system (6) in order to saturate the homoclinic instability and thus to carry out the reinjection process in the neighborhood of the origin avoiding the trajectories diverging to infinity.

2.2 Simulation. - In the sequel of this paper, we will describe some numerical investigations of the Poincaré map model (6) following a one-parameter path in the space of constraints defined by the straight line :

$$
\nu=\nu^{*}+17.897\left(\mu-\mu^{*}\right)
$$

with

$$
\mu^{*}=1.705 \times 10^{-4}, \quad \nu^{*}=3.046 \times 10^{-3}
$$

and $\eta=5 \times 10^{-2}, \quad k_{1}=1.4, \quad k_{2}=-2.12 \times 10^{3}$, $k_{3}=0, k_{4}=-48, k_{5}=-1.392 \times 10^{-6}$. This path goes through (at least within our numerical resolution) the fuzzy region previously discussed [30]. To simplify the notation, we will consider $\varepsilon=\mu-\mu^{*}$ as the control parameter (in fact locally it is proportional to the $\varepsilon$ involved in the Hopf normal form (1)). In order to check the continuous character of the transition along the path (11), we have estimated the hysteresis range (if any) at the transition to chaos to be less than $\Delta \varepsilon=10^{-7}$.

In figure 1 , we illustrate the type-II intermittency scenario observed along the one-parameter path (11). For negative values of $\varepsilon\left(\mu<\mu^{*}\right)$, the origin $x=y=z=0$ is asymptotically stable. When crossing the critical value $\varepsilon=0\left(\mu=\mu^{*}, \nu=\nu^{*}\right)$, the system loses its regularity and chaotic bursts interrupt episodes of laminar behavior in an apparently random fashion. Immediatly beyond the threshold of intermittency $(\ln (\varepsilon)=-13)$, the laminar phases are long and only very exceptional bursts emerge occasionally as seen in figure 1a. Slightly above criticality $(\ln (\varepsilon)=-11)$, the chaotic episodes become more frequent and as seen in figures $1 \mathrm{~b}$ and $1 \mathrm{c}$, the oscillatory behavior of the coordinate $z$ during the laminar episodes corresponds to a nonsaturated modulation of the amplitude of the original periodic oscillation of the periodically forced oscillator (5) as
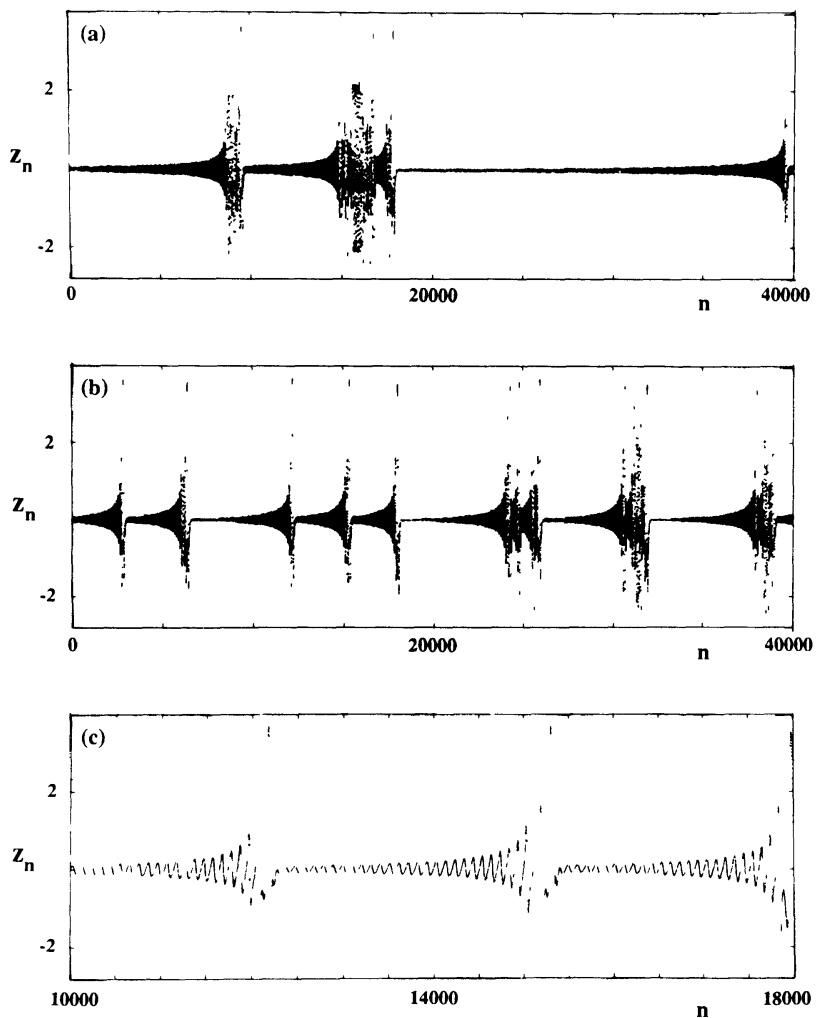

Fig. 1. $-z$ (in $\delta^{5}$ units, with $\delta=5 \times 10^{-2}$ ) versus the number $n$ of iterates as computed with the Poincare map model (6) for the parameter values given in equation (11). (a) $\quad \mu=1.728 \times 10^{-4}, \quad \nu=3.087 \times 10^{-3}$; $\mu=1.875 \times 10^{-4}, \nu=3.349 \times 10^{-3}$; (c) is the same as (b) but with a smaller range of $n$-values. 
observed in reference [16]. When the amplitude of this modulation reaches some critical value, then the dynamics changes drastically and a turbulent burst is initiated. Immediately after the chaotic intermission there is a reappearance of the regular behavior, the length of which depends on the distance of the reinjection point to the origin.

In figure 2a, we illustrate a two-dimensional projection of the dynamics onto the unstable manifold of the origin. Only a few iterates of the mapping (6) have been retained in order to distinguish both the one-dimensional homoclinic reinjection mechanism and the very smooth spiraling behavior away from the origin which is the laminar episode. The reinjection distribution as projected onto the unstable manifold of the origin is shown as the histogram in figure $2 b$. The whole set of reentry points does not spread out on such a surface but they instead fall approximately along a curve as expected from a homoclinic reinjection process $[16,26,27]$. $\dot{A}$ similar histogram has been obtained in reference [16] with the periodically driven third-order nonlinear oscillator (5) ; it reflects the numerical finding that the Poincaré map of a homoclinic strange attractor is a multifolded line (line $\times$ Cantor) where the folds are generally strongly transversally contracted. Therefore, this histogram shows a clear evidence that the chaotic reinjection process is essentially one-dimensional and that consequently it breaks the rotational invariance of the Hopf normal form (1). But this symmetry is at the heart of the Pomeau-Manneville [1] analysis. Therefore we need to revise the theoretical predictions (2), (3) and (4) in order to bring them into accord with the onedimensional deterministic reinjection mechanism in the mapping (6).

If one assumes a white radial reentry distribution $\left(0<\rho<R_{\mathrm{r}}\right): P_{\mathrm{r}}\left(\rho_{\mathrm{r}}\right)=1 / R_{\mathrm{r}}\left(\theta_{\mathrm{r}}=\theta^{*}\right)$, then the probability distribution $P_{\ell}(n)$ of the laminar episodes is predicted to scale like $n^{-3 / 2}$ at small laminar period $n$ and to decay exponentially $P_{\ell}(n) \sim$ $\exp (-2 \varepsilon n)$ at large $n$. This distribution [31] is characterized by a mean value :

$$
\begin{aligned}
&\langle n\rangle=1 /(2 \varepsilon)\left\{\log \left[\left(1+\varepsilon / a R_{\mathrm{r}}^{2}\right) /\left(1+\varepsilon / a R_{\ell}^{2}\right)\right]+\right. \\
&\left.+2\left(\varepsilon / a R_{\mathrm{r}}^{2}\right)^{1 / 2} \operatorname{atan}\left[\left(a R_{\mathrm{r}}^{2} / \varepsilon\right)^{1 / 2}\right]\right\}
\end{aligned}
$$

where $R_{\mathrm{r}}$ is distinguished from $R_{\ell}$ in order to match our numerical situation where $R_{\mathrm{r}}$ is found much smaller than the cut-off $R_{\ell}$ which defines the laminar episodes. Then the scaling law (3) transforms into

$$
\langle n\rangle \sim \varepsilon^{-1 / 2} \text { if } \varepsilon \ll a R_{\mathrm{r}}^{2}
$$

while the behavior (4) at larger values of $\varepsilon$ is unchanged :

$$
\langle n\rangle \sim \ln (\varepsilon) / \varepsilon \quad \text { if } \quad \varepsilon \gg a R_{\mathrm{r}}^{2} .
$$

Figure 3 provides confirmation to these theoretical predictions; the average length of the laminar periods is plotted versus the control parameter $\varepsilon$. We have considered $10^{5}$ laminar periods as defined by $R_{\mathrm{r}}=5 \times 10^{-2}$ and $R_{\ell}=5 \times 10^{-1}$. The cross-over between the scaling behaviors (13) and (14) is clearly shown to occur for $\ln (\varepsilon) \sim-12$.

A typical characteristic of intermittencies is the $1 / f^{\Delta}$ divergencies in the small frequency limit of the spectra which reflects the arbitrarily long laminar regions observed immediately beyond criticality. Because of the one-dimensional character of the homoclinic process [31], some of the results obtained with one-dimensional map models [32-34] are expected to apply to type-II intermittency as simulated with the three-dimensional Poincaré map model (6).
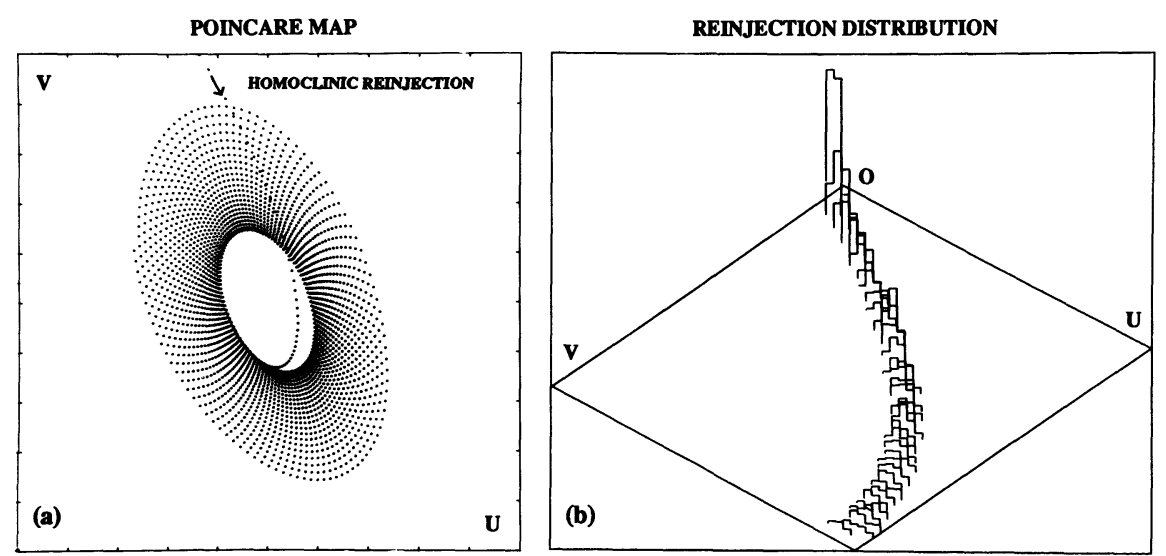

Fig. 2. - (a) Projection of the Poincaré map onto the local two-dimensional unstable manifold of the origin. Only a finite set of iterates of the mapping (6) are considered in order to visualize the homoclinic reinjection and a spiraling-out laminar episode. (b) Histogram of the reinjection distribution inside the local two-dimensional unstable manifold of the origin $\mathrm{O}$. Same model parameters as in figure $1 \mathrm{~b}$. 


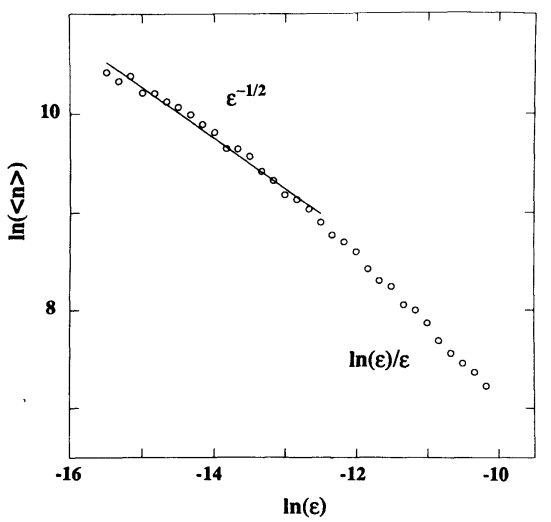

Fig. 3. - The average length of $10^{5}$ laminar episodes $\langle n\rangle$ versus $\varepsilon$ in $\log$-log scales. The model parameters are given in equation (11). The solid line corresponds to the theoretical prediction (13). The laminar episodes are defined by $R_{\mathrm{r}}=5 \times 10^{-2}$ and $R_{\ell}=5 \times 10^{-1}$.

The numerical power spectrum presented in figure 4 seems to corroborate this feature : the exponent $\Delta=0.63 \pm 0.10$ extracted from a mean square fit of the power spectrum $v s$. the frequency in log-log scale is quite compatible with the logarithmic corrections to a $1 / f^{1 / 2}$ divergence predicted in reference [33]. This power spectrum was computed from $2^{3}$ samples of $2^{9}$ iterates of the mapping (6) using a Hamming window. Let us note, however, that the departure

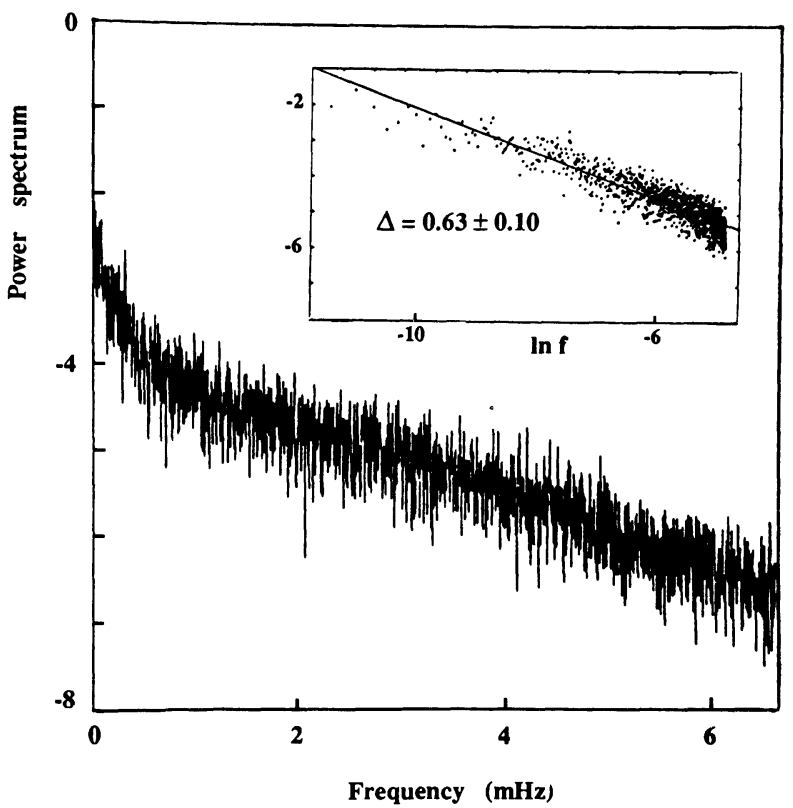

Fig. 4. - The power spectrum as computed using the Hamming windowing procedure from $2^{3}$ samples of $2^{9}$ iterates of the Poincare map model (6) with the parameter values given in equation (11) and $\mu=1.709 \times 10^{-4}, \nu=3.052 \times 10^{-3}(\ln (\varepsilon)=-15)$. The time between two iterates of the mapping (6) has been normalized to one second. In the insert, the power spectrum is plotted versus $\ln (f)$. from the exponent $\Delta=1 / 2$ (which has been observed in the periodically driven oscillator (5) as well [16]) can also be attributed to the fact that our numerical experiment has been conducted at small but finite distance from the intermittency threshold [13].

\section{Type-II intermittency in the presence of noise : numerical results.}

When observations are compared with theory, it is essential to understand the role of external noise. During the last few years, considerable progress have been accomplished in understanding the effect of stochastic (as well as periodic) perturbations on systems which exhibit a continuous transition to chaos [35]. For type-I intermittency, the instability of the scenario $[14,35]$ to the presence of an external noise has been investigated numerically $[2,36]$ and theoretically [37]. Renormalization group techniques have been used $[3,38-40]$ to predict the scaling behavior of the order parameter, i.e. the envelope of the largest Lyapunov exponent $(\bar{L})$ or the inverse of the average length of the laminar episodes $\left(\langle n\rangle^{-1}\right)$, as a function of the amplitude of the perturbation. To conclude this paper we extend this study to typeII intermittency. In fact we will limit ourselves to a numerical review of different situations corresponding to the insertion of additive and multiplicative noises in the Poincaré map model (6). Although we will discuss only the numerical results obtained with the three-dimensional discrete system (6), we have also performed subsequent simulations of different kinds of stochastic forcing of the two-dimensional normal form (1) of the subcritical Hopf bifurcation with random uniformly distributed reentry points. Let us mention that these simulations corroborate the scaling behaviors observed when iterating the Poincaré map model (6) in the presence of noise.

3.1 AdDITIVE NOISE. - Figure 5a illustrates the effect of additive random noise as modeled by the introduction of the term $h \xi$ in the third equation in the Poincaré map model (6) :

$$
\left\{\begin{aligned}
x^{\prime}= & x+y \\
y^{\prime}= & y+z \\
z^{\prime}= & (1-\eta) z-\nu y-\mu x-k_{1} x^{2}-k_{2} y^{2}- \\
& \quad-k_{3} x y-k_{4} x z-k_{5} x^{2} z+h \xi
\end{aligned}\right.
$$

where $\xi$ is a Gaussian random variable, with $\langle\xi\rangle=0$ and $\left\langle\xi_{n}, \xi_{n^{\prime}}\right\rangle=\delta_{n n^{\prime}} ; h$ is a variable that controls the width (or amplitude) of the noise. Different values of $\varepsilon=\left(\mu-\mu^{*}\right)$ have been investigated along the numerical path (11); the values presented in figure 5a correspond to $\ln (\varepsilon)=-12$, -13 and -14 respectively and belong to the range of $\varepsilon$ values where the inverse of the order parameter 

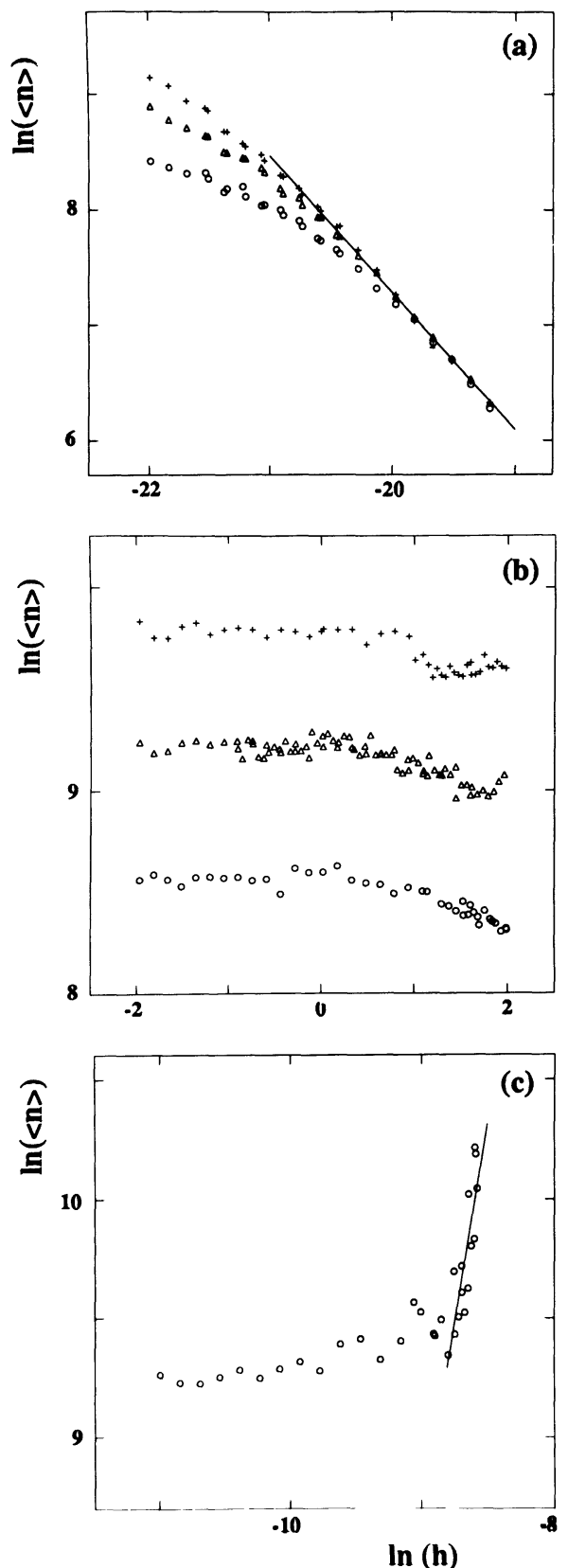

Fig. 5. - The average value $\langle n\rangle$ of the laminar episodes versus the amplitude of the noise $h$ in log-log scales. The model parameters are defined in equation (11). Several values of $\varepsilon$ are considered $: \ln (\varepsilon)=-12$ (circles) $;-13$ (triangles); - 14 (crosses). (a) Additive noise : equation (15), the solid line corresponds to a linear regression fit with an exponent $\alpha=-1.19 \pm 0.05 ;$ (b) Multiplicative noise : equation (16); (c) Multiplicative noise : equation (17), the solid line is drawn to guide the eyes.

scales like $\langle n\rangle \sim \varepsilon^{-1 / 2}$ as predicted by equation (13) and shown in figure 3. Above some cut-off values $h_{\mathrm{c}}(\varepsilon)$, the noise becomes more important than the deterministic drift obtained beyond criticality and the average length of the laminar episodes $\langle n\rangle$ decreases when one strengthens the noise. This indicates that the intermittency threshold occurs for $\varepsilon<0$, i.e. the transition to chaos is advanced in the presence of additive noise. In a log-log plot, a linear regression fit yields an exponent $\alpha=-1.19 \pm$ 0.05 , which is very likely to correspond to a linear increase of the order parameter $\langle n\rangle^{-1} \sim \bar{L} \sim h$ up to some logarithmic corrections. These observations clearly illustrate the sensitivity of type-II intermittency to the presence of additive noise. The instability of this scenario is also characterized by a shift of the cross-over point which seems compatible with a square-root power-law in $\varepsilon$ up to some logarithmic corrections. Let us mention that we have obtained the same quantitative measurement when considering the addition of random noise in the (subcritical) Hopf normal form (1).

3.2 Multiplicative NOISE. - Figure 5b illustrates the influence of a multiplicative noise as introduced through the coefficient $k_{1}^{\xi}=k_{1}+h \xi$ of the nonlinear term $x^{2}$ in the Poincare map model (6)

$$
\left\{\begin{aligned}
x^{\prime}= & x+y \\
y^{\prime}= & y+z \\
z^{\prime}= & (1-\eta) z-\nu y-\mu x-\left(k_{1}+h \xi\right) x^{2}- \\
& -k_{2} y^{2}-k_{3} x y-k_{4} x z-k_{5} x^{2} z .
\end{aligned}\right.
$$

We consider values of $h$ such that $h x^{2}$ covers the same range of values as the amplitude $(h)$ of the additive noise in figure $5 \mathrm{a}$. For the three values of $\varepsilon$ previously investigated, $\langle n\rangle$ does not seem to be affected (at least at leading order) by this particular multiplicative noise; subsequent computations strongly suggest that type-II intermittency is stable with respect to the presence of fluctuations in the coefficients $k_{i}$ of the nonlinear terms in the threedimensional mapping (6). This statement is further corroborated by direct simulations of the Hopf normal form (1) in the presence of stochastic fluctuations in the coefficient $a$ of the cubic nonlinear term in the modulus equation.

Figure $5 \mathrm{c}$ shows the case where the noise is assigned to the control parameter $\mu^{\xi}=\mu+h \xi$ :

$$
\left\{\begin{aligned}
x^{\prime}= & x+y \\
y^{\prime}= & y+z \\
z^{\prime}= & (1-\eta) z-\nu y-(\mu+h \xi) x-k_{1} x^{2}- \\
& \quad-k_{2} y^{2}-k_{3} x y-k_{4} x z-k_{5} x^{2} z
\end{aligned}\right.
$$

Unlike additive noise, beyond some cut-off value $h_{\mathrm{c}}(\varepsilon)$, the noise begins to affect the dynamics but in such a way that $\langle n\rangle$ increases with $h$. This effect, which is also encountered when investigating the Hopf normal form (1) with a noisy control parameter $\varepsilon$, suggests that this time, the intermittency threshold is very likely to be postponed $(\varepsilon=$ $\left.\mu-\mu^{*}>0\right)$ by the noise. Unfortunately, because 
in this case the study of the statistics of the laminar periods becomes increasingly time consuming, we have not been able to extract the power law behavior (if any) of $\langle n\rangle$ as a function of the amplitude of the noise.

This numerical analysis is only the prelude to a theoretical understanding of the effect of a random noise on type-II intermittency. The reduction of the Poincare map model (6) in the presence of noise to a noisy Hopf normal form using stochastic centermanifold and normal form techniques [41, 42] would be a major step toward this goal. However, this reduction would require to adapt these perturbative techniques to discrete noisy systems which, to our knowledge, is not yet established in the literature. Then with further approximation of this stochastic two-dimensional map by a Focker-Planck equation $[2,37,43-45]$, a quantitative analysis should be attainable. As a first theoretical attempt [46], we have used the axial symmetry of the Hopf normal form (1) to reduce this study to the analysis of a onedimensional Focker-Planck equation. Within such a working hypothesis, we have been able to understand some of our numerical results [46] e.g. the exponent $\alpha=-1$ extracted from the iteration of the discrete system (15). However, this study is still preliminary and incomplete since it does not take into account corrections which are very likely to be induced by some breaking of the axial symmetry as observed in our simulations. We hope to elaborate on this point in a forthcoming communication.

\section{Acknowledgments.}

We are very grateful to $H$. P. Herzel for very interesting discussions and to L. Tuckerman for a careful reading of the manuscript.

\section{References}

[1] Manneville, P. and Pomeau, Y., Phys. Lett. A 75 (1979) 1 ;

Pomeau, Y. and Manneville, P., Commun. Math. Phys. 74 (1980) 189.

[2] Hirsch, J. E., Huberman, B. A. and Scalapino, D. J., Phys. Rev. A 25 (1982) 519.

[3] Hu, B. and Rudnick, J., Phys. Rev. Lett. 48 (1982) 1645.

[4] Manneville, P., Phys. Lett. A 79 (1980) 33.

[5] Scholz, H. J., Yamada, T., Brand, H. and Graham, R., Phys. Lett. A 82 (1981) 321.

[6] Meunier, C., Bussac, M. N. and Laval, G., Physica D 4 (1982) 236.

[7] Berge, P., Dubois, M., Manneville, P. and Pomeau, Y., J. Phys. France Lett. 41 (1980) L341.

[8] Maurer, J. and Libchaber, A., J. Phys. France Lett. 41 (1980) L-515.

[9] Pomeau, Y., Roux, J. C., Rossi, A., Bachelart, S. and VIDAL, C., J. Phys. France Lett. 42 (1981) L-271.

[10] Jeffries, C. and Perez, J., Phys. Rev. A 26 (1982) 2117.

[11] Dubois, M., Rubio, M. A. and Berge, P., Phys. Rev. Lett. 51 (1983) 1446.

[12] Tresser, C., Coullet, P. and Arneodo, A., J. Phys. France Lett. 41 (1980) L-243.

[13] Arneodo, A., Kreisberg, N., MCCormick, W. D., RichetTI, P. and Swinney, H. L., in preparation.

[14] Collet, P., Coullet, P. and Tresser, C., J. Phys. France Lett. 46 (1985) L-143.

[15] Iooss, G., Bifurcation of Maps and Applications (North-Holland, Amsterdam 1979), Chapter III.

[16] Richetti, P., Argoul, F. and Arneodo, A., Phys. Rev. A 34 (1986) 726.
[17] Marsden, J. E. and McCraken, M., Hopf Bifurcation and its Applications, Appl. Math. Sci. (Springer, N.Y.) 1976, 19.

[18] Iooss, G. and JosEPH, D. D., Elementary Stability and Bifurcation Theory (Springer, N.Y.) 1980, Chapters VII and VIII.

[19] Guckenheimer, J. and Holmes, P., Nonlinear Oscillations, Dynamical Systems and Bifurcations of Vector Fields (Springer, N.Y.) 1984, Chapter 3.

[20] ShiL'Nikov, L. P., Sov. Math. Dokl. 6 (1965) 163 ; Math. USSR Sb. 6 (1968) 427 and 10 (1970) 91.

[21] Arneodo, A., Coullet, P. H. and Spiegel, E. A., Phys. Lett. A 94 (1983) 1.

[22] Arneodo, A., Coullet, P. H., Spiegel, E. A. and Tresser, C., Physica D 14 (1985) 327.

[23] Iooss, G., Chaos and Statistical Mechanics, Ed. Y. Kuramoto, Springer Ser. in Synergetics (1984).

[24] Argoul, F. and Arneodo, A., J. Mech. Th. \& Appl., numéro spécial (1984) p. 241.

[25] ARnold, V. I., Geometrical Methods in the Theory of Ordinary Differential Equations (Springer, N.Y.) 1983.

[26] Gaspard, P. and Nicolis, G., J. Stat. Phys. 31 (1983) 499.

[27] Gaspard, P., mémoire de Licence, University of Brussels (1982), unpublished.

[28] Rossler, O. E., Phys. Lett. A 71 (1979) 155.

[29] Smale, S., Bull. Am. Math. Soc., 6 (1967) 803.

[30] Such a path for the Poincaré map model (6) corresponds to an investigation of equation (8) with parameter values $\bar{\mu}, \bar{\nu}, \bar{\eta}, k_{i}$ of order unity and $\delta \cdot=5 \times 10^{-2}$.

[31] Note that with this one-dimensional reinjection process, the distribution of laminar episodes for type-II intermittency becomes similar to the distribution for type-III intermittency. 
[32] Manneville, P., J. Phys. France 41 (1980) 1235.

[33] Ben-Mizrachi, A., Procaccia, I., Rosenberg, N., SChMidt, A. and Schuster, H. G., Phys. Rev. A 31 (1985) 1830.

[34] So, B. C. and Mori, H., Physica D 21 (1986) 126.

[35] Argoul, F. and Arneodo, A., Lect. Notes Math. 1186 (1986) 338 and references therein.

[36] Mayer-Kress, G. and Haken, H., Phys. Lett. A 82 (1981) 151.

[37] Eckmann, J. P., Thomas, L. and Wittwer, P., $J$. Phys. A 14 (1981) 3153.

[38] Hirsch, J. E., Nauenberg, M. and Scalapino, D. J., Phys. Lett. A 87 (1982) 391.

[39] Argoul, F. and Arneodo, A., J. Phys. France Lett. 46 (1985) L-901.

[40] Argoul, F., Arneodo, A., Collet, P. and Lesne, A., Europhys. Lett. 3 (1987) 643.
[41] Knobloch, E. and Wiesenfeld, K. A., J. Stat. Phys. 33 (1983) 611.

[42] Coullet, P. H., Elphick, C. and Tirapegui, E., Phys. Lett. A 111 (1985) 277.

[43] Stratonovich, R. L., Topics in the Theory of Random Noise, 1 (Gordon and Breach, New York) 1963, Chapter 5.

[44] Graham, R., in Quantum Statistics in Optics and Solid State Physics, 66 Springer Tracts in Mod. Phys. (Springer, Berlin) 1973, Chapter 8.

[45] Horsthemke, W. and LefeVer, R., Noise-Induced Transitions : Theory and Applications in Physics, Chemistry and Biology, Springer Ser. in Synergetics (Springer, Berlin) 15 (1984) Chapter 4.

[46] Argoul, F., Arneodo, A. and Hertzel, H. P., in preparation. 